\title{
Risk-adjusted morbidity and mortality models to compare the performance of two units after major lung resections
}

\author{
Alessandro Brunelli, MD, ${ }^{a}$ Nicholas J. Morgan-Hughes, MD, ${ }^{\mathrm{b}}$ Majed Refai, MD, ${ }^{\mathrm{a}}$ Michele Salati, MD, \\ Armando Sabbatini, MD, and Gaetano Rocco, MD ${ }^{\mathrm{c}}$
}

Objective: We sought to develop risk-adjusted morbidity and mortality models to compare the performance of 2 different thoracic surgery units in patients submitted to major lung resections.

Methods: Seven hundred forty-three patients (551 male and 192 female patients) who underwent lobectomy $(\mathrm{n}=611)$ or pneumonectomy $(\mathrm{n}=132)$ from January 2000 through August 2004 at 2 European thoracic units (519 patients in unit A and 224 patients in unit B) were analyzed. Risk-adjusted models of 30-day or in-hospital cardiopulmonary morbidity and mortality were developed by using stepwise logistic regression analyses and validated by means of bootstrap analysis. Preoperative and operative variables were initially screened by using univariate analysis. Those with a $P$ value of less than .10 were used as independent variables in the regression analyses. The regression equations were then used to estimate the risk of outcome, and the observed and predicted outcome rates of the 2 units were compared by using the $z$ test for comparison of proportions.

Results: The following regression models were developed. Predicted morbidity: $\ln R / 1-R=-2.4+0.03$ Xage $-0.02 X_{p p o F E} V_{1}+0.6$ Xcardiac comorbidity (Hosmer-Lemeshow statistic $=6.1[P=.6], \mathrm{c}$ index $=0.65$ ). Predicted mortality: $\ln R 1-R=-6.97+0.095 X a g e-0.042 X_{p p o F E V}$ (Hosmer-Lemeshow statistic $=2.99[P=.9], \mathrm{c}$ index $=0.77)$. The models proved to be stable at bootstrap analyses. No differences were noted between observed and predicted outcome rates within each unit, despite an apparent unadjusted better performance of unit B.

From the Unit of Thoracic Surgery, Umberto I ${ }^{\circ}$ Regional Hospital, ${ }^{a}$ Ancona, Italy; the Department of Anaesthesiology, Sheffield Teaching Hospital, ${ }^{\text {b }}$ Sheffield, United Kingdom; and the Division of Thoracic Surgery, National Cancer Institute, ${ }^{\mathrm{c}}$ Pascale Foundation, Naples, Italy.

Read at the Eighty-sixth Annual Meeting of The American Association for Thoracic Surgery, Philadelphia, Pa, April 29-May 3, 2006.

Received for publication April 22, 2006; revisions received Aug 13, 2006; accepted for publication Aug 31, 2006.

Address for reprints: Alessandro Brunelli, MD, Via S Margherita 23, Ancona 60129, Italy (E-mail: alexit_2000@yahoo.com).

J Thorac Cardiovasc Surg 2007;133:88-96

$0022-5223 / \$ 32.00$

Copyright $\odot 2007$ by The American Association for Thoracic Surgery

doi:10.1016/j.jtcvs.2006.08.058
Conclusions: The use of risk-adjusted outcome models avoided misleading information derived from the unadjusted analysis of performance. Risk modeling is essential for the evaluation of the quality of care.

I n the present era of managed care systems, increasing pressure is exerted by payers and the public on the profession to assume greater responsibility for delivering high-quality care. Accountability demands an increased effort in quality-monitoring and quality-improvement processes through the analysis of performance of health care providers. However, because patients are not randomly allocated to different physicians or hospitals and their outcome can be influenced by illness severity, treatment effectiveness, or even mere chance, ${ }^{1,2}$ the comparison of outcomes is challenging. Indeed, a different case mix at different institutions might make the comparison of crude outcome rates misleading. Therefore the selection of quality end points must account for the differences in the prevalence of risk factors, and clinical risk modeling must become the logical and necessary approach for provider profiling. Nevertheless, risk modeling for audit purposes appears yet in its embryonic phase in thoracic surgery, and specific literature on this issue is scanty and mainly limited to internal audit. ${ }^{3-5}$ 


\section{Abbreviations and Acronyms \\ DLCO = carbon monoxide lung diffusion capacity \\ $\mathrm{FEV}_{1} \quad=$ forced expiratory volume in 1 second \\ ppoDLCO $=$ predicted postoperative carbon monoxide lung diffusion capacity \\ $\operatorname{ppoFEV}_{1}=$ predicted postoperative forced expiratory volume in 1 second \\ $\mathrm{Vo}_{2} \max =$ maximum oxygen consumption}

The objective of the present study was to develop riskadjusted models of early morbidity and mortality after major lung resection and to compare the performance of 2 different European thoracic surgery units with the intent to provide a methodological and practical example of model building and application for multicentric comparative audit purposes.

\section{Patients and Methods}

We analyzed 743 patients treated with lobectomy/bilobectomy (n $=611)$ or pneumonectomy $(\mathrm{n}=132)$ in 2 European centers $(519$ in center A and 224 in center B) from January 2000 through August 2004.

This is an observational study performed on prospective, periodically audited electronic databases of two dedicated thoracic surgery units located in two different European countries. Data were entered prospectively in each database by a trained staff physician and were periodically audited by a designated audit lead, who was responsible for the accuracy and completeness of the database. Both databases are used as continuous quality-improvement instruments at the two participating units. The study was approved by the local institutional review board of each center, and informed consent to be entered in the databases was obtained from all patients.

The patients and datasets used for this study were the same used for a recently published analysis. ${ }^{6}$ Although the subject was similar (risk modeling), the focuses of the two analyses were different in that the first study aimed at demonstrating the superiority of bootstrap analysis over the traditional training and test method for developing risk models by constructing multiple models from the population from unit $\mathrm{A}$ and then assessing their validity on the bootstrapped population of unit B. ${ }^{6}$ No comparison between the two units was performed because this was not the objective of that report. On the other hand, in this analysis the main purpose was to compare the performance of the two units by developing risk models from the entire dataset of patients (unit A plus unit B) after validation by means of bootstrap analysis. For this reason, we consider the two studies unique and independent.

In both centers surgical intervention was contraindicated in those patients with a predicted postoperative forced expiratory volume in 1 second $\left(\mathrm{ppoFEV}_{1}\right)$ and predicted postoperative carbon monoxide lung diffusion capacity (ppoDtco) of less than 30\% of predicted value in association with a poor exercise capacity (height at maximal stair-climbing test $<12 \mathrm{~m}$ or maximum oxygen consumption $\left[\mathrm{VO}_{2} \mathrm{max}\right]$ at cycle ergospirometry $<10 \mathrm{~mL} \cdot \mathrm{kg}^{-1}$ $\mathrm{min}^{-1}$ ) or in the presence of hemodynamic instability, despite optimization of treatment. As a rule, lung resections were per- formed through a muscle-sparing thoracotomy by certified thoracic surgeons for benign (56 lobectomies and 2 pneumonectomies) or malignant (555 lobectomies and 130 pneumonectomies; 667 primary and 18 metastatic diseases) diseases. The postoperative management policies were the same in both centers. All patients were admitted to a dedicated general thoracic surgery ward immediately after the operation, resorting to the intensive care unit only in case of complications requiring invasive assisted ventilation or invasive continuous monitoring. Postoperative treatment was standardized in both units and focused on early mobilization, chest physiotherapy and physical rehabilitation, thoracotomy pain control, and antibiotic and antithrombotic prophylaxis. Postoperative chest pain was controlled by means of epidural or continuous intravenous analgesia, which was titrated to keep the pain visual analogue score at less than 4 (in a scale ranging from 0-10) for the first postoperative 48 to 72 hours (pain score was assessed twice daily during the morning and afternoon rounds).

Postoperative morbidity and mortality were considered as those occurring within 30 days postoperatively or for a longer period if the patient was still in the hospital.

A number of preoperative and operative variables were tested for possible association with outcome variables (see Appendix $1^{4,5,7-9}$ for explanation of variables).

Data were initially scrutinized for assessing the quality of variables and their consistency in definition and recording between the 2 units. To this purpose, the 2 databases were reciprocally and independently audited by the principle investigator of the other unit $(\mathrm{AB}$ and GR). Only those variables and end points that were deemed of high quality and consistent across the 2 units were included in this analysis. The databases were made anonymous for both patients and surgeons and were merged for analysis. All patients were initially used to develop the predictive logistic models. For each measure of outcome (morbidity and mortality rates), variables were initially screened by using univariate analyses. The univariate comparisons of outcomes were performed by means of the unpaired Student $t$ test for numeric variables with normal distribution and by means of the Mann-Whitney $U$ test for numeric variables without normal distribution. The Shapiro-Wilk normality test was used to assess normal distribution. Categoric variables were compared by means of the $\chi^{2}$ test or the Fisher exact test, as appropriate.

Variables with a $P$ value of less than .10 at univariate analysis were then used as independent variables in the stepwise logistic regression analyses. The presence or absence of 1 or more complications or of mortality was used as a dependent variable in each respective model. All data were complete, with the exception of data on carbon monoxide lung diffusion capacity (DLCo), which were $95 \%$ complete. Missing data were imputed by averaging the nonmissing values. Potential explanatory variables more than $5 \%$ incomplete were excluded from this analysis (ergometric parameters $\left[\mathrm{VO}_{2} \mathrm{max}\right]$, blood gas analysis measures, and albumin concentration). To avoid multicollinearity, only 1 variable in a set of variables with a correlation coefficient of greater than 0.5 was selected (by using the bootstrap procedure) and used in the regression model.

A $P$ value of less than .05 was selected for retention of variables in the final model. The area under the receiver operating characteristic curve or $\mathrm{c}$ index was used to study the discrimination ability of each model. Hosmer-Lemeshow goodness-of-fit statis- 
TABLE 1. Characteristics of the patients in the study (aggregate and by center)

\begin{tabular}{|c|c|c|c|c|}
\hline Variables & $\begin{array}{l}\text { All patients } \\
\text { ( } n=743)\end{array}$ & $\begin{array}{c}\text { Unit A } \\
(n=519)\end{array}$ & $\begin{array}{c}\text { Unit B } \\
(n=224)\end{array}$ & $\begin{array}{c}\text { Unit } A \text { vs unit } B, \\
P \text { value }\end{array}$ \\
\hline Age (y) & $65.2(10.7)$ & $66.9(9.6)$ & $61.3(12.2)$ & $<.0001^{*}$ \\
\hline Elderly (>70 years of age; $n[\%]$ ) & $275(37)$ & $220(42)$ & $55(25)$ & $<.0001 \dagger$ \\
\hline BMI $\left(\mathrm{kg} / \mathrm{m}^{2}\right)$ & $25.9(4.4)$ & $26(4.4)$ & $25.6(4.5)$ & $.3^{*}$ \\
\hline $\mathrm{FEV}_{1} \%$ & $84.7(18.9)$ & $85(19.3)$ & $84(18.2)$ & $.4^{*}$ \\
\hline Dıco\% & $78(18.8)$ & $76.5(18.6)$ & $81.4(18.2)$ & $.005^{*}$ \\
\hline ppoFEV $_{1} \%$ & $65.5(17.3)$ & $66.9(16.9)$ & $62.4(17.8)$ & $.001^{*}$ \\
\hline ppoDıco\% & $60.4(16.9)$ & $60.3(16.8)$ & $60.6(16.4)$ & $.5^{*}$ \\
\hline Cardiac comorbidity (n [\%]) & $287(39)$ & $253(49)$ & $34(15)$ & $<.0001 \dagger$ \\
\hline Neoadjuvant chemotherapy (n [\%]) & $85(11)$ & $60(12)$ & $25(11)$ & $0.9 \dagger$ \\
\hline Pneumonectomy (n [\%]) & $132(18)$ & $90(17)$ & $42(19)$ & $0.6 \dagger$ \\
\hline
\end{tabular}

Results are expressed as means (standard deviations) unless otherwise indicated. $B M I$, Body mass index; $F E V_{1}$, forced expiratory volume in 1 second; $D\llcorner C O$, carbon monoxide lung diffusion capacity; ppoFEV ${ }_{1}$, predicted postoperative forced expiratory volume in 1 second; ppoDLco, predicted postoperative carbon monoxide lung diffusion capacity. *Mann-Whitney Test. $\dagger \chi^{2}$ Test.

tics were used to assess the calibration of the models. Furthermore, the multivariate procedures were validated by means of bootstrap bagging with 1000 samples. In the bootstrap procedure repeated samples of the same number of observations as the original database $(n=743)$ were selected with replacement from the original set of observations. For each sample, stepwise logistic regression was performed, entering the variables with a $P$ value of less than .1 at univariate analysis. The stability of the final model can be assessed by identifying the variables that enter most frequently in the repeated bootstrap models and comparing those variables with the variables in the final model. If the final stepwise model variables occur in a majority $(>50 \%)$ of the bootstrap models, the original final stepwise regression model can be judged to be stable. ${ }^{6,10,11}$ We have previously shown that bootstrap analysis might be particularly advantageous in moderate-sized samples inasmuch as it allows the use of the entire dataset for model developing without the need to split and further reduce the sample size (and the number of outcome cases). ${ }^{6}$
The logistic models were then used to predict morbidity and mortality in the patients undergoing operations in the 2 different units. Predicted and observed outcome rates in each unit were then compared, and $P$ values were calculated from the $z$ test statistic for the difference between two proportions, which uses the sampling distribution of the statistic to guess population parameters. In the test statistic the numerator is the difference between the proportion in the two samples, and the denominator is the standard error of the difference in the two proportions.

All the statistical tests were two-tailed. The analysis was performed with STATA 8.2 (Stata Corp, College Station, Tex) statistical software

\section{Results}

The characteristics of the patients in the study are shown in Table 1.

Table 2 shows the results of the univariate comparison

TABLE 2. Univariate comparison between patients with and without complications and between dead and surviving patients

\begin{tabular}{|c|c|c|c|c|c|c|}
\hline Variables & $\begin{array}{c}\text { Complicated } \\
\text { (n = 159) }\end{array}$ & $\begin{array}{c}\text { Noncomplicated } \\
(\mathrm{n}=584)\end{array}$ & $P$ value & $\begin{array}{c}\text { Dead } \\
(n=35)\end{array}$ & $\begin{array}{l}\text { Surviving } \\
\text { (n = 708) }\end{array}$ & $P$ value \\
\hline Age & $68(10.2)$ & $64.4(10.7)$ & $<.0001^{*}$ & $71.5(6.7)$ & $64.9(10.8)$ & $.0001^{*}$ \\
\hline Male sex (n [\%]) & $132(83)$ & $419(72)$ & $.004 \uparrow$ & $30(86)$ & $521(74)$ & $.12 \ddagger$ \\
\hline BMI $\left(\mathrm{kg} / \mathrm{m}^{2}\right)$ & $26.3(4.1)$ & $25.8(4.5)$ & $.2^{*}$ & $25.2(4.4)$ & $25.9(4.4)$ & $.4^{*}$ \\
\hline $\mathrm{FEV}_{1} \%$ & $80.3(17.3)$ & $85.9(19.2)$ & $.0009^{*}$ & $74.9(16.3)$ & $85.2(19)$ & $.002^{*}$ \\
\hline Dıco\% & $74.8(19.6)$ & $78.9(18.3)$ & $.02^{*}$ & $71.3(23)$ & $78.3(18.3)$ & $.05^{*}$ \\
\hline $\mathrm{ppoFEV}_{1} \%$ & $62.1(15.9)$ & $66.5(17.5)$ & $.003^{*}$ & $56.1(12.7)$ & $66(17.3)$ & $.0004^{*}$ \\
\hline ppoDıco\% & $57.4(15.6)$ & $61.2(16.9)$ & $.03^{*}$ & $53.5(18)$ & $60.7(16.6)$ & $.01^{*}$ \\
\hline Malignant disease (n [\%]) & $152(96)$ & $533(91)$ & $0.07 \dagger$ & $33(94)$ & $652(92)$ & $1 \ddagger$ \\
\hline Cardiac comorbidity (n [\%]) & $84(53)$ & $203(35)$ & $<.0001 \dagger$ & $20(57)$ & 267 (38) & $.02 \dagger$ \\
\hline Neoadjuvant chemotherapy (n [\%]) & $24(15)$ & $61(10)$ & $.1 \dagger$ & $4(11)$ & $81(11)$ & $1 \ddagger$ \\
\hline Pneumonectomy (n [\%]) & $32(20)$ & $100(17)$ & $.4 \dagger$ & $12(34)$ & $120(17)$ & $.009 \dagger$ \\
\hline
\end{tabular}

Results are expressed as means (standard deviations) unless otherwise indicated. BMI, Body mass index; FEV ${ }_{1}$, forced expiratory volume in 1 second; $D L C O$, carbon monoxide lung diffusion capacity; ppoFEV ${ }_{1}$, predicted postoperative forced expiratory volume in 1 second; ppoDLCO, predicted postoperative carbon monoxide lung diffusion capacity. *Mann-Whitney test. $\dagger \chi^{2}$ Test. $\ddagger$ Fisher exact test. 
TABLE 3. Results of the stepwise logistic regression analyses (dependent variables: morbidity and mortality, respectively): Parsimonious models

\begin{tabular}{|c|c|c|c|c|c|c|}
\hline Predictors & Coefficients & SE & Odds ratio & $95 \% \mathrm{CL}$ & $P$ value & Bootstrap frequency \\
\hline \multicolumn{7}{|l|}{ Morbidity model } \\
\hline Intercept & -2.4 & & & & & \\
\hline Age & 0.03 & 0.01 & 1.03 & $1.009-1.05$ & .005 & $80 \%$ \\
\hline ppoFEV $_{1} \%$ & -0.02 & 0.006 & 0.98 & $0.97-0.99$ & .003 & $88 \%$ \\
\hline Cardiac comorbidity & 0.6 & 0.2 & 1.8 & $1.2-2.6$ & .002 & $89 \%$ \\
\hline \multicolumn{7}{|l|}{ Mortality model } \\
\hline Intercept & -6.97 & & & & & \\
\hline Age & 0.095 & 0.03 & 1.1 & $1.05-1.16$ & .0002 & $100 \%$ \\
\hline ppoFEV $_{1} \%$ & -0.042 & 0.01 & 0.96 & $0.94-0.98$ & .0004 & $98 \%$ \\
\hline
\end{tabular}

Bootstrap frequency: frequency of significance $(P<.05)$ in 1000 bootstrap samples. $S E$, Standard error; $C L$, confidence limit; $p p o F E V_{1}$, predicted postoperative forced expiratory volume in 1 second.

between patients with and without complications or mortality. In particular, compared with noncomplicated patients, those with complications were older $(P<.0001)$; had lower forced expiratory volume in 1 second $\left(\mathrm{FEV}_{1} ; P=.0009\right)$, Dlco $(P=.02), \operatorname{ppoFEV}_{1}(P=.003)$, and ppoDlco $(P=$ $.03)$ values; were more frequently male $(P=.004)$; and had a higher prevalence of malignant disease $(P=.07)$ and cardiac comorbidities $(P<.0001)$.

Compared with patients who survived, those who died were older $(P=.0001)$; had lower $\operatorname{FEV}_{1}(P=.002)$, DLco $(P=.05), \operatorname{ppoFEV}_{1}(P=.0004)$, and ppoDlco $(P=.01)$ values; and had a higher prevalence of cardiac comorbidities $(P=.02)$ and pneumonectomies $(P=.009)$.

Stepwise logistic regression analysis showed that significant and reliable predictors of morbidity were age $(P=$ $.005), \operatorname{ppoFEV}_{1}(P=.003)$, and cardiac comorbidity $(P=$ .002 , Table 3).

The following equation predicting morbidity was developed:

$\ln R / 1-R=-2.4+0.03$ Xage $-0.02 X_{\text {ppoFE }}$

$$
+0.6 \text { Xardiac comorbidity }
$$

(Hosmer-Lemeshow statistic $=6.1[P=.6], \mathrm{c}$ index $=$ $0.65)$. Stepwise logistic regression analysis showed that significant and reliable predictors of mortality were age $(P$ $=.0002)$ and $\operatorname{ppoFEV}_{1}(P=.0004$, Table 3$)$.

The following equation predicting mortality was developed:

$$
\ln R / 1-R=-6.97+0.095 \text { Xage }-0.042 X_{p p o F E V}
$$

(Hosmer-Lemeshow-statistic $=2.99[P=.9], \mathrm{c}$ index $=$ $0.77)$. Figure 1 plots the cumulative observed morbidity and mortality rates against the predicted rates in patients ordered by increasing risk of morbidity and mortality, respectively. The plots showed overall good calibration of the models.

The stability of the models was evaluated by assessing the distribution of the $\mathrm{c}$ index statistics across 1000 boot- strap samples. The morbidity model $\mathrm{c}$ index was greater than 0.6 in $99 \%$ of the samples and greater than 0.7 in $46 \%$ of the samples. The mortality model $\mathrm{c}$ index was greater than 0.6 in $100 \%$ of the samples and greater than 0.7 in $97 \%$ of the samples.
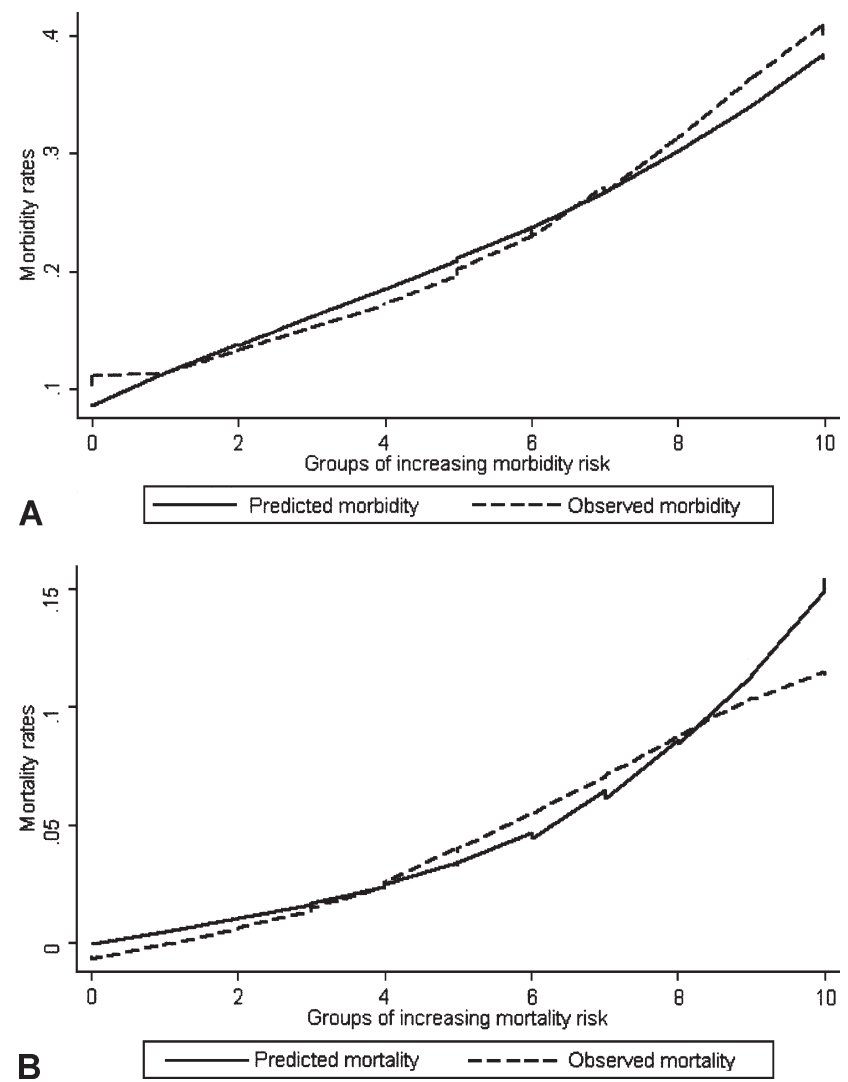

Figure 1. A, Plot of cumulative predicted morbidity against observed morbidity ordered by increasing risk. B, Plot of cumulative predicted mortality against observed mortality ordered by increasing risk. 

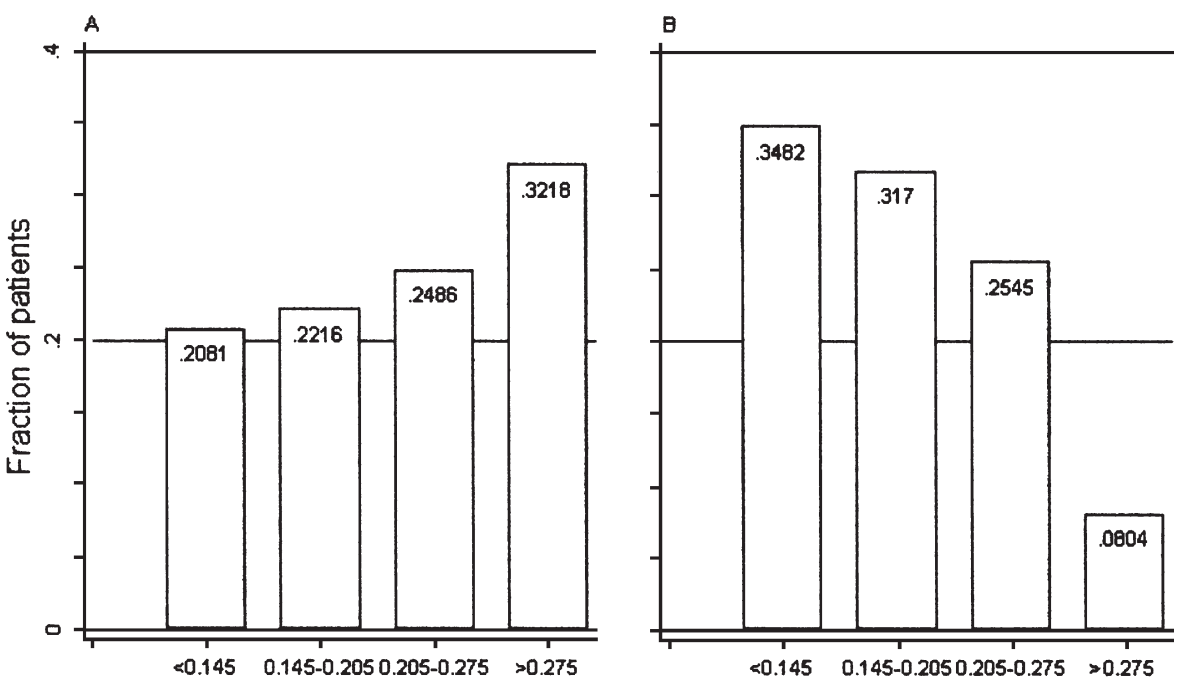

A

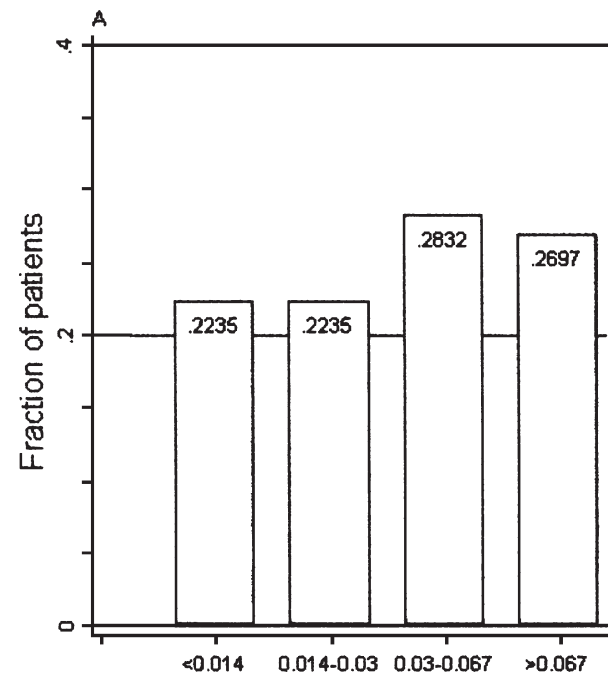

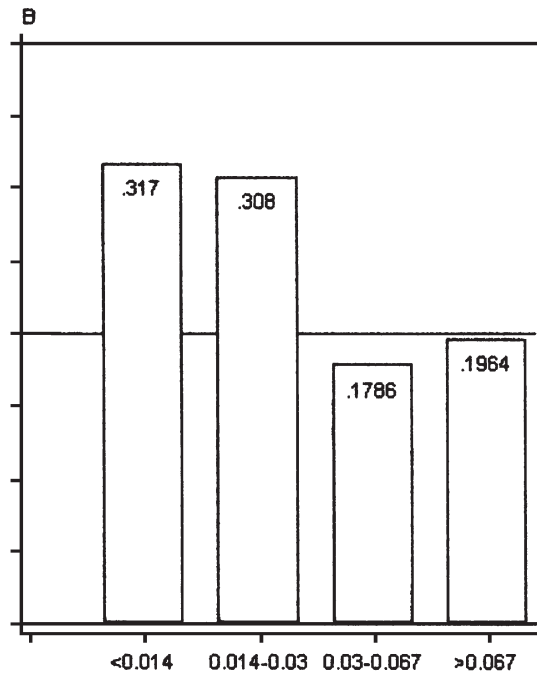

Figure 2. A, Distribution of quartiles of predicted risk of morbidity in the 2 units. B, Distribution of quartiles of predicted risk of mortality in the 2 units.
We observed differences in baseline and operative characteristics between patients in unit $\mathrm{A}$ and those in unit B. In particular, compared with patients in unit B, those operated on in unit A were older $(P<.0001)$, had lower DLco values $(P=.005)$, and had a greater frequency of cardiac comorbidities but had higher ppoFEV ${ }_{1}$ values $(P=.001$, Table 1$)$.

Figure 2 depicts the distributions of the patients according to increasing quartiles of expected risk of morbidity and mortality in the 2 units, respectively. Unit A had a greater frequency of patients at higher risk of morbidity $(P<$ $.0001)$ and mortality $(P=.001)$ compared with unit B.

Observed morbidity and mortality rates were 23\% (120 patients) and $4.8 \%$ (25 patients) in unit $\mathrm{A}$ and $17 \%$ (39 patients) and $4.4 \%$ (10 patients) in unit B, respectively.

The comparison of predicted and observed outcomes is shown in Table 4. Despite a higher observed morbidity rate in unit $\mathrm{A}(P=.07)$, no differences were noted between observed and predicted outcomes rates in each unit.

\section{Discussion}

In the era of accountability, a reliable analysis of performance of health care providers is essential. Because patients are not randomized between different hospitals and great variations can occur because of referral and geographic patterns, the comparison of outcomes among different centers might be challenging.

Two recent, important multi-institutional articles from the American College of Surgeons Oncology Group and the American College of Surgeons Commission on Cancer were aimed at setting modern outcome benchmarks and patterns of care in our specialty. Yet by reporting very different crude outcome figures after major pulmonary resections, 
TABLE 4. Comparison of predicted and observed morbidity and mortality rates within each unit

\begin{tabular}{|c|c|c|c|c|c|c|}
\hline \multirow[b]{2}{*}{ Unit } & \multicolumn{2}{|c|}{ Morbidity } & \multirow[b]{2}{*}{$P$ value } & \multicolumn{2}{|c|}{ Mortality } & \multirow[b]{2}{*}{$P$ value } \\
\hline & Observed & Predicted & & Observed & Predicted & \\
\hline Unit A & $23 \%$ & $22.7 \%(22 \%-23.5 \%)$ & .9 & $4.8 \%$ & $4.9 \%(4.5 \%-5.3 \%)$ & .9 \\
\hline Unit B & $17 \% *$ & $18.2 \%(17 \%-19 \%)$ & .7 & $4.4 \% \dagger$ & $4.2 \%(3.6 \%-4.9 \%)$ & .9 \\
\hline
\end{tabular}

Expected outcomes are presented with $95 \%$ confidence limits. $* P=.07$, unit $\mathrm{A}$ versus unit $\mathrm{B} . \dagger P=.8$, unit $\mathrm{A}$ versus unit $\mathrm{B}$.

likely reflecting different eligibility criteria, they emphasize the need for risk adjustment. ${ }^{12,13}$

The quality end points must be necessarily risk adjusted to account for differences in patients' baseline and operative characteristics. In fact, crude outcome rates might lead to inappropriate clinical and administrative decisions and cause unethical risk-averse behaviors.

As a consequence, risk modeling should become an integral part of any quality-monitoring and quality-improvement program. In our specialty risk stratification for audit purposes is still in its embryonic phase, and only few experiences have been published on this issue..$^{3-5}$ In particular, examples of risk-adjusted multicentric comparative analysis of performance are lacking.

Recently, the European Association for Cardiothoracic Surgery/European Society of Thoracic Surgeons European Thoracic Database project produced a model of in-hospital mortality from a dataset of more than 3400 lung resections collected voluntarily from 27 units in 14 countries over a period of 3 years. $^{7}$ This work represented the first multiinstitutional multinational effort to develop an objective instrument to analyze the performance of different units across Europe. Future developments of the project are expected to refine the preliminary model and set performance benchmarks in Europe.

The present study must be interpreted as an example of methods and application of risk models for provider-initiated multicentric comparative audit purposes. In fact, it was conceived to develop risk-adjusted morbidity and mortality models to compare the performance of two different thoracic surgery units. Because no model is better than the one derived from the data at hand and because it has been shown that ready-made models applied to external populations perform less well than internally derived models, ${ }^{2,14-16}$ we elected not to use existing external models. Furthermore, it is known that regression models perform better when applied retrospectively to evaluate the past performance. ${ }^{17}$ Under a total qualitymanagement perspective, they are not meant to foretell the future but to analyze past data to avoid repeating problems encountered in the past. ${ }^{17}$ In this regard the retrospective application of a model as a diagnostic quality instrument to the data from which it was developed seems justified, provided a cross-sample validation (bootstrap) had been performed to measure its reliability. ${ }^{6,10,11}$
In surgical practice morbidity and mortality are the most commonly used clinical indicators of quality. When used as an outcome variable, however, complications have inherent problems: their definition might be complex and subjective, and their recording might vary among different institutions and even within the same unit during successive periods of time. In this work complications were prospectively and independently recorded at two different centers after strict criteria were preliminarily defined. It was our priority to assess the consistency of these definitions between the two centers, and only those complications that were judged to be reliably consistent in definition and recording were used for the analysis (see Appendix 1 for definition of variables). Furthermore, the two main investigators (AB and GR) are member of the European Thoracic Database Committee ${ }^{7}$ and share the same methods and purposes in variable definition and database quality control. They were designated as the clinical audit leads responsible for periodically verifying the quality of the databases.

The same issue applied for the selection of the variables tested for a possible association with outcomes. Only those high-quality variables that were at least $95 \%$ complete in each database and were deemed to be consistent between the two units were selected and used for the analysis. Variables that were more than $5 \%$ incomplete, such as ergometric parameters $\left(\mathrm{VO}_{2} \max \right)$, blood gas analysis measures, and albumin concentration, were not included in the analysis. We are aware that our models might be imperfect and subject to improvements in terms of individual discrimination by the addition of other important factors associated with postoperative outcome (ie, ergometric parameters), but they are presumably the most reliable ones that we could derive in the context of this analysis. They showed a good face value and content validity and had a good predictive validity, as assessed by using the bootstrap bagging simulation. Our models are parsimonious enough to obviate overfitting problems when applied to medium-sized populations (particularly when the events are rare, such as mortality). It has recently been shown that increasing the number of predictors does not necessarily improve the discrimination of the models. ${ }^{18}$ Conversely, keeping the models as parsimonious as possible might be attractive because it can prevent many problems: cost of data collection, errors and imprecision in data recording, missing val- 
ues, and instability of the model. The ideal model should be based on clinical, high-quality, prospectively compiled, periodically audited, specialty-specific, and procedure-specific (lung resection) databases. We think our models met these criteria.

Bootstrapping was used to validate the models once they were developed from the entire cohort of patients. We and others have shown that this method is superior to the traditional training and test splitting of the dataset, inasmuch as more reliable and reproducible predictive equations are generated. ${ }^{6,10}$ Each model was tested on 1000 bootstrap samples of the same number of patients as the original dataset, and only reliable predictors were selected and factored into the final regression equations.

As ever, caution is required in interpreting the prediction of a risk model in an individual patient. The individual discrimination of the models (c statistics) was moderate but in line with the ones reported in other studies. ${ }^{5,8}$ This common finding in surgical models might be partly due to yet unknown predictors, to the difficulty to represent complex clinical conditions or pathways of care with one or more variables, and to catastrophic random events that are rare in the population but important for the single patients. ${ }^{19}$ However, the models could be reliably applied to the whole population of lung resection candidates as audit instruments.

Another important issue central to every audit analysis is the definition of quality of care, which most likely is the reflection of the entire process of care rather than of a single outcome end point. However, in the absence of more precise instruments to evaluate the quality of care in its wholeness, multiple end points should be analyzed as a surrogate ${ }^{20}$ because each end point might be associated with a different aspect of the quality of care. In this regard our selected indicators (morbidity and mortality) are only a few of the multiple end points that could be risk adjusted and used for audit purposes (eg, postoperative stay, intensive care unit admission, technical complications, readmission rate, longterm survival, quality of life, and residual functional state).

After development and validation, our models were applied for predicting the outcomes in the two units, and they were able to prevent misleading information derived from the unadjusted analysis of the performance. In fact, despite unit $\mathrm{A}$ having a higher observed morbidity rate compared with that of unit B, the observed outcome rates were in line with the predicted ones in each unit. The increased observed morbidity in unit A could be explained by a worse physiologic state of the patients at the time of operation rather than by a poorer performance, as shown also by the higher frequency of patients with higher predicted morbidity and mortality risks in unit A compared with those in unit B. Without the use of risk adjustment, unit A would have been erroneously regarded as underperforming unit B.
It must be noted that our models were designed for audit purposes only and were not meant to be used for patient selection, a process that should be based more on individual clinical evaluation rather than on a population-based risk model. $^{21}$

This work confirmed that risk modeling is essential for provider profiling and can be easily used for a fair comparison of the performance between different centers, with the ultimate goal of improving the quality of surgical care. The costs for implementing and managing international multicentric databases (eg, the European Thoracic Surgery Database or the Society of Thoracic Surgeons thoracic database) seem therefore justified by the benefits that could derive from the qualityimprovement processes that will be based on them. Even though start-up costs might be daunting, ultimately, improved quality will be cost-efficient, and part of any cost savings realized by improved quality can be even factored into the total costs of gathering and maintaining risk-adjusted data. We think that important international cooperative processes for monitoring and standardization of the pathways of surgical care and for the accreditation of structures cannot leave out of consideration the use of reliable risk-adjustment models. As physicians, we should assume complete responsibility in the evaluation of our performance. We should not let managers and administrators judge our practice through imprecise and improper instruments. At a minimum, it would be in our best interest to provide them with the right evaluation tools, which must necessarily take into account our proficiency in clinical risk adjustment.

\section{References}

1. Iezzoni LI. The risks of risk adjustment. JAMA. 1997;278:1600-7.

2. Luft HS, Romano PS. Chance, continuity, and change in hospital mortality rates. Coronary artery bypass graft patients in California hospitals, 1993 to 1998. JAMA. 1993;270:331-7.

3. Brunelli A, Fianchini A, Al Refai M, Gesuita R, Carle F. Internal comparative audit in a thoracic surgery unit using the physiological and operative severity score for the enumeration of mortality and morbidity (POSSUM). Eur J Cardiothorac Surg. 2001;19:924-8.

4. Brunelli A, Fianchini A, Al Refai M, Salati M. A model for the internal evaluation of the quality of care after lung resection in the elderly. Eur J Cardiothorac Surg. 2004;25:884-9.

5. Brunelli A, Xiumé F, Al Refai M, Salati M, Marasco R, Sabbatini A. Risk-adjusted morbidity, mortality and failure-to-rescue models for internal provider profiling after major lung resection. Interact Cardiovasc Thorac Surg. 2006;5:92-6.

6. Brunelli A, Rocco G. Internal validation of risk models in lung resection surgery: bootstrap versus training and test sampling. $J$ Thorac Cardiovasc Surg. 2006;131:1243-7.

7. Berrisford R, Brunelli A, Rocco G, Treasure T, Utley M, on behalf of the Audit and Guidelines Committee of the European Association for Cardiothoracic Surgery and the European Society of Thoracic Surgeons. The European Thoracic Surgery Database project: modelling the risk of in-hospital death following lung resection. Eur J Cardiothorac Surg. 2005;28:306-11.

8. Harpole DH Jr, DeCamp MM Jr, Daley, Hur K, Oprian CA, Henderson WG, Khuri SF. Prognostic models of thirty-day mortality and morbidity after major pulmonary resection. J Thorac Cardiovasc Surg. 1999;117:969-79. 
9. Ferguson MK, Durkin AE. A comparison of three scoring systems for predicting complications after major lung resection. Eur J Cardiothorac Surg. 2003;23:35-42.

10. Blackstone EH. Breaking down barriers: helpful breakthrough statistical methods you need to understand better. J Thorac Cardiovasc Surg. 2001;122:430-9.

11. Grunkemeier GL, Wu YX. Bootstrap resampling method: something for nothing? Ann Thorac Surg. 2004;1142-4.

12. Little AG, Rush VW, Bonner JA, Gaspar LE, Green MR, Webb WR, et al. Patterns of surgical care of lung cancer patients. Ann Thorac Surg. 2005;80:2051-6.

13. Allen MS, Darling GE, Pechet TT, Mitchell JD, Herndon JE, Landreneau RJ, et al. Morbidity and mortality of major pulmonary resections in patients with early-stage lung cancer: initial results of the randomised prospective ACOSOG Z0030 trial. Ann Thorac Surg. 2006;81: 1013-20.

14. Ivanov J, Tu JV, Naylor CD. Ready-made, recalibrated or remodeled? Issues in the use of risk indexes for assessing mortality after coronary artery bypass graft surgery. Circulation. 1999;99:2098-104.

15. DeLong ER, Peterson ED, DeLong DM, Muhlbaier LH, Hackett S, Mark DB. Comparing risk-adjustment methods for provider profiling. Stat Med. 1997;16:2645-64.

16. Orr RK, Naini BS, Sottile FD, Dumas EM, O'Mara P. A comparison of four severity-adjusted models to predict mortality after coronary artery bypass graft surgery. Arch Surg. 1995;130:301-6.

17. Chassin MK, Hannan EL, DeBuono BA. Benefits and hazards of reporting medical outcome publicly. N Engl J Med. 1996;334:394-8.

18. Tu JV, Sykora K, Naylor CD. Assessing the outcome of coronary artery bypass graft surgery: how many risk factors are enough? Steering Committee of the cardiac Care Network of Ontario. J Am Coll Cardiol. 1997;30:1317-23.

19. Shahian DM, Blackstone EH, Edwards FH, Grover FL, Grunkemeier GL, Naftel DC, et al. Cardiac surgery risk models: a position article. Report from the STS workforce on evidence-based surgery. Ann Thorac Surg. 2004;78:1868-77.

20. Silber JH, Rosenbaum PR, Schwartz JS, Ross RN, Williams SV. Evaluation of the complication rate as a measure of quality of care in coronary artery bypass graft surgery. JAMA. 1995;274:317-23.

21. Esteva H, Marchevsky A, Nunez T, Luna C, Esteva M. Neural networks as a prognostic tool of surgical risk in lung resections. Ann Thorac Surg. 2002;73:1576-81.

\section{Appendix 1}

\section{Preoperative and Operative Variables}

The following variables were initially screened for a possible association with postoperative morbidity and mortality: age, sex, body mass index (BMI; in kilograms per square meter), $\mathrm{FEV}_{1}$, DLco, ppoFEV1, ppoDlco, cardiac comorbidity, type of disease (benign vs malignant), type of operation (lobectomy vs pneumonectomy), and neodjuvant chemotherapy.

Pulmonary function tests were performed according to the American Thoracic Society criteria. Results of spirometry were collected after bronchodilator administration. DLco measurement was performed by using the single-breath method.

$\mathrm{FEV}_{1}$, ppoFEV $_{1}$, DLco, and ppoDlco values were expressed as percentages of predicted value for age, sex, and height. ppoFEV and ppoDLCO values were calculated by estimating the amount of functioning parenchyma removed during operation by means of bronchoscopy, computed tomography, and quantitative lung perfusion.

For the purpose of the present study and in accordance with previous investigations, ${ }^{4,5}$ a concomitant cardiac disease was defined as follows: previous cardiac surgery, previous myocardial infarction, history of coronary artery disease, and current treatment for arrhythmia, cardiac failure, or hypertension. We chose to use this definition of cardiac comorbidity for the sake of comparison with previous studies and for numeric reasons. In fact, breaking down the variable in the single cardiac diseases would have resulted in too many cofactors with limited representation. Although not weighed, all cardiac conditions included in the variable are widely recognized cardiac risk factors for noncardiac surgery.

\section{Outcome Variables}

For the purpose of this study, according to previous studies ${ }^{5,8,9}$ and to the European Association for Cardiothoracic Surgery/ European Society of Thoracic Surgeons thoracic surgery database, ${ }^{7}$ the following complications were included: respiratory failure requiring mechanical ventilation for more than 48 hours, pneumonia (chest radiographic infiltrates, increased white blood cell count, and fever), atelectasis requiring bronchoscopy, adult respiratory distress syndrome, pulmonary edema, pulmonary embolism, myocardial infarction (suggestive electrocardiographic findings and increased myocardial enzymes), hemodynamically unstable arrhythmia requiring medical treatment, cardiac failure (suggestive chest radiographs, physical examination, and symptoms), acute renal failure (change in serum creatinine level $>2$ $\mathrm{mg} / \mathrm{dL}$ compared with preoperative values), and stroke. For numeric reason, we did not separate cardiac and pulmonary complications. We also did not weigh complications in keeping with most of the work done on morbidity; however, we included only those complications that increased the complexity of postoperative management, requiring new treatments or a change of treatment, therefore adding up to hospital costs and stay.

\section{Discussion}

Dr Mark S. Allen (Rochester, Minn). Dr Yang, Dr Sonett, members, and guests. I have no conflicts to disclose other than that I am a member of the Society of Thoracic Surgeons Database Committee, and therefore my encouragement to get you to join is hereby noted.

The authors are to be congratulated on an excellent presentation on a topic that is becoming increasingly important in the field of health care, and especially surgery. The push for quality improvement and the means to measure what good quality is has already become an important part of our daily practice and can only be expected to increase in importance in the future. Analyses such as these using data with risk adjustment are very important so that we can accurately assess the progress we are making in improving the quality of care we deliver to our patients. This analysis of more than 700 patients who underwent operations in two separate European hospitals is a good example of how we, the surgeons, should lead the effort so that it can be done in a scientific manner that is valid and meaningful.

I have several questions for the author. In your report you used a model to predict the number of complications. Because there is obviously a difference between someone with symptomatic postoperative atrial fibrillation that is easily controlled with medication versus someone with adult respiratory distress syndrome that requires prolonged ventilatory support and intensive care unit care, were you able to dissect out from your data the risk factors for specific complications, or was there insignificant statistical power to provide for these types of specific predictions? 
Second, the question that always arises in the United States is whether a board-certified thoracic surgeon has a better outcome than a nonthoracic board-certified surgeon when performing a pulmonary resection. Do you have the ability in your dataset to answer this question using these European data? Were all of these procedures performed by certified thoracic surgeons?

Finally, your statistical analysis is quite complicated, and I am certainly not a statistical expert; however, I am concerned about the relatively small sample size you have. With only 743 patients and 35 deaths in your analysis, that leaves the standard error for age at plus or minus about $30 \%$ of the risk coefficient for age and plus or minus $25 \%$ of the coefficient for the percent predicted postoperative $\mathrm{FEV}_{1}$. Did your statistician give you an estimate of how many additional patients you would need to obtain a lower standard of error, say, a plus or minus $5 \%$. In other words, how large of a database do we need to accumulate to get a more specific predictive number?

Again, I appreciate your efforts to provide us with stratification in the field of pulmonary surgery and appreciate the opportunity to discuss this article. Thank you.

Dr Brunelli. Thank you, Dr Allen, for your kind comments and your questions.

As to the first question, the complications, we did not weight the complications, and therefore each complication was accounted for similarly, and I think we did not have the numbers to individually dissect the complications, for example, to make separate models for pulmonary and cardiac complications. Therefore, the numbers and the statistical power precluded us from making this subgroup analysis.

You asked about the qualifications of the surgeons. These two centers were dedicated thoracic surgery units, and all of the surgeons were qualified, certified thoracic surgeons. Therefore we could not assess the differences between credentials of surgeons in relation to outcome.

Finally, sample size is a major concern in our specialty when dealing with audit analysis. These are the numbers. Of course, 35 deaths seems to be a low number, but we are confident that the results are reliable. We used bootstrap analysis to validate the model. The mortality model had two predictors, and therefore it is not overfitted, and the predictors had high stability with bootstrap analysis, which means that we repeated the regression analysis in 1000 new simulated samples drawn with replacement from the original dataset, and these variables turned out to be significant in more than $85 \%$ or $90 \%$ of cases. It took us 5 years to aggregate these data, and these two units have the average volume load in Europe, and this is a major concern. Even if you aggregate multiple units, let us say 10, 20, or 100 units, when dealing with audit and comparison between the units, we have to then apply the models to the single units or to the single surgeon and then you can have problems. Perhaps we have to shift from outcome measures to other measures of quality of care, perhaps process measures, which are less affected by random noise and numbers and perhaps more linked to the quality. Therefore, the main purpose of this study was to provide an example or method, and of course there are limitations, but I think the limitations are inherent to the outcome measures selected. For this first audit, we wanted to do a comparative audit between two thoracic surgery units using the two most commonly used outcome measures, which are morbidity and mortality. But of course sample size is very important, and perhaps we can change our mentality and shift from outcome measures to process measures.

Dr Stephen C. Yang (Baltimore, Md). I am less of a statistician than Dr Allen, but how would you see the results to be different if there was a significant difference in mortality rates or complications between the two institutions? Would this model still hold?

Dr Brunelli. There was no significant difference-

Dr Yang. I know, but what if?

Dr Brunelli. Oh, what if? You have to assess whether there is a difference in the predicted and observed mortality within the units. Therefore let us say we have a big difference in mortality rate, observed mortality rate between the two units. If the units were not in line with the predicted values, you can be confident that the difference is not due to poor performance but perhaps a different case mix.

Dr Joshua R. Sonett (New York, NY). But dead is dead in the end, and it does not matter what your risk model is if you have very bad outcomes. If it is much higher, then maybe that is a reflection of patient selection, which must be considered when evaluating programs and surgeons.

Dr Brunelli. Yes, but we are dealing with audit analysis and performance evaluation. Therefore it is unfair to judge a unit on crude mortality rate without taking into account the case mix and the prevalence of risk factors.

Dr Yang. Well, even if the case mixes are different, what about the experience of the surgeons? Are they fairly well balanced between the 2 units?

Dr Brunelli. Yes. We explored this, and the structural and organizational characteristics of the two units were remarkably similar in credentials, experience of the surgeons, nurse-to-patient ratio, intensive care unit policy, and management. They both were dedicated thoracic surgery wards. We were confident that the qualifications and experience of the surgeons and the structure of the units were similar. 